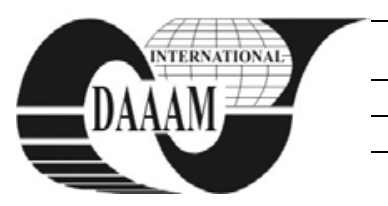

\title{
HILL'S MUSCLE MODEL-BASED MODELING OF PNEUMATIC ARTIFICIAL MUSCLE
}

\author{
HOSOVSKY, A[lexander] \& HAVRAN, M[ichal]
}

\begin{abstract}
The paper presents pneumatic artificial muscle modeling based on the modified Hill's muscle model. Hill's model is used for modeling a mechanical part of the system whereas Boyle-Mariott's ideal gas law is used for deriving pressure differential equation. The model is expected to be a part of PAM-based actuator model with two PAMs designed for industrial applications.
\end{abstract}

Key words: artificial muscle, Hill's model, spring, damper, force

\section{INTRODUCTION}

Modeling of pneumatic artificial muscles (PAM) and PAMbased devices was subject of several papers published so far (Kerscher,2007). Some of them were based on the Schulte's results, where the basic formula for a muscle force was derived based on the energy conservation principle and geometric parameters of a muscle. Experiments in these works were carried out using common McKibben muscles, the braiding of which was separated from the tube. The contact between the braiding and the tube causes force-length relationship to be hysteretic what was confirmed in our previous research (Boržíková,2009). In (Kerscher,2007;Serres,2008) Fluidic muscles (which use different construction with less pronounced hysteresis) were used for experiments. Based on our previous findings we decided to use Fluidic muscles as well. Our model is based on the knowledge gained throughout the research with all unique features associated with intended application as well as with utilized components, available theory about the processes taking place within this system and some results obtained by other research teams (Židek,2010). The model itself is divided into two main parts: mechanical and pneumatic.

As a part of previous research, static modeling for a pneumatic artificial muscle was carried out. This has to be improved to include also dynamic properties so that the dynamic model of the actuator can be derived.

The muscle generates two kinds of forces : active and passive, sum of which constitutes the total muscle force. Socalled contractile element is the source of active force (Fig.1). The passive force in this model is provided by two nonlinear springs, one parallel to contractile element, the other one in series with it. These springs are the source of restoring forces tending to return the muscle to its original

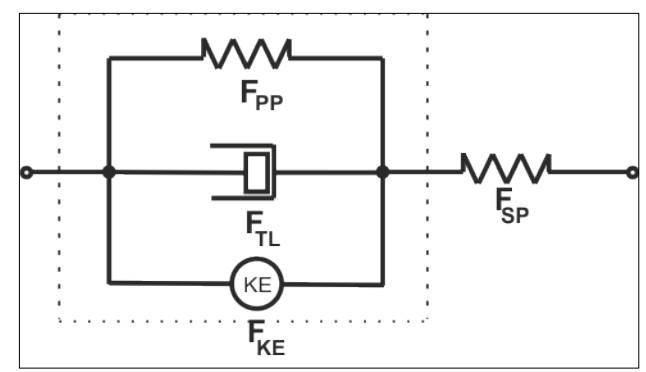

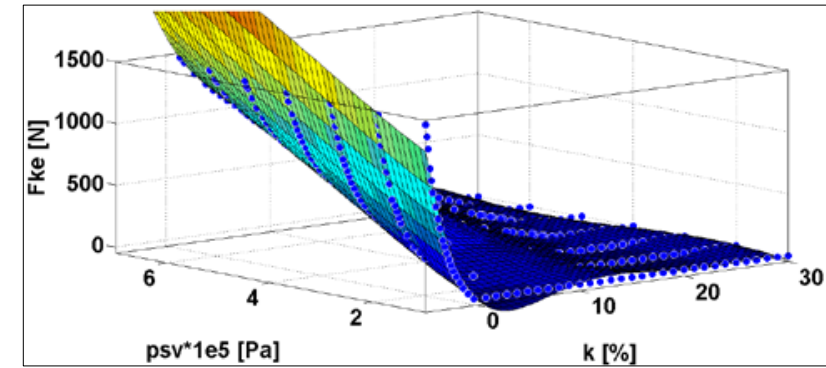

Fig. 2. Approximated function $\left(F_{K E}=f\left(k, P_{S V}\right)\right.$

length. Only the part marked with dashed line in Fig.1 is considered for our model. The force of a paralel nonlinear spring is a force needed for compressing the tube during contraction and it decreases the active force of contractile element (Shadmehr,2005).

Using Newton's second law we can write :

$$
m \cdot \frac{d^{2} y}{d t^{2}}+F_{T L}+F_{P P}=F_{K E}-F_{E}
$$

where $m$ - moving mass, $y$ - muscle displacement, $F_{T L}-$ damper force, $F_{P P}$ - paralel spring force, $F_{K E}$ - contractile element force, $F_{E}$ - external force. The force of contractile element is a nonlinear function of muscle pressure and contraction $\left(F_{K E}=f\left(k, P_{S V}\right)\right)$. This relationship was approximated using fifth-order polynomial with 21 coefficients. The coefficients were determined using Surface Fitting Tool in Matlab's Curve Fitting Toolbox. The resulting surface is depicted in Fig.2. Muscles used in experiments were fitted with force compensator which limits generated force to $1200 \mathrm{~N}$. The range of this function was thus constrained to the interval $F_{K E} \in\langle 0,1200\rangle$.

We used 3-position 2-way on/off valves with closed center (MATRIX EMX821.104C224). The manufacturer indicates two parameters in his datasheet: critical ratio $b=0,433$ and sonic conductance $C=2,6167 \cdot 10^{-9} \mathrm{~m}^{3} \cdot \mathrm{s}^{-1} \cdot \mathrm{Pa}^{-1}$. In (Beater,2007) formulas for modeling mass flow through a valve which have become ISO standard 6358 are specified. They have been rewritten to a volume flow form:

$$
\dot{V}_{V Z}=\left\{\begin{array}{c}
P_{1} \cdot C \cdot \sqrt{\frac{T_{0}}{T_{1}}} \cdot \sqrt{1-\left(\frac{\frac{P_{2}}{P_{1}}-b}{1-b}\right)^{2}}, \text { if } \frac{P_{2}}{P_{1}}>b \\
P_{1} \cdot C \cdot \sqrt{\frac{T_{0}}{T_{1}}}, \text { if } \frac{P_{2}}{P_{1}} \leq b
\end{array}\right.
$$

where $\dot{V}_{V Z}$ - volume flow $\left[\mathrm{m}^{3} . \mathrm{s}^{-1}\right], P_{1}$ - absolute upstream pressure $[\mathrm{Pa}], C_{-}$sonic conductance $\left[\mathrm{m}^{3} \cdot \mathrm{s}^{-1} \cdot \mathrm{Pa}^{-1}\right], T_{0}-$ temperature at reference conditions $[\mathrm{K}], T_{1}$ - upstream temperature $[\mathrm{K}], P_{2}-$ absolute downstream pressure $[\mathrm{Pa}]$. The time rate of change of muscle pressure can be derived using Boyle-Mariott's law for ideal gases with multiplication of pressure and volume being constant (Kerscher,2007):

Fig. 1. Hill's muscle model 


$$
\dot{P}_{S V}=P_{A} \frac{\dot{V}_{V Z}}{V_{S V}}-P_{S V} \frac{\dot{V}_{S V}}{V_{S V}}
$$

where $\dot{V}_{V Z}$ is time derivative of air volume in muscle (volume flow through the valve) and $\dot{V}_{S V}$ is time derivative of muscle volume. The volume flow through the valve is defined by 2 and it is needed to add expressions for the calculation of muscle volume and its derivative based on its geoemtric parameters.

\section{RESULTS}

All parts of the resulting model were implemented separately in Simulink and then integrated to a final model. The time step of the simulation was set to a fixed value of $0,0001 \mathrm{~s}$. ODE8 Dormand-Prince continuous solver was used for experiments. At the time of writing, the only variable that could be compared to a variable of a real muscle (for model validation) was muscle pressure. The pressure in muscle was measured using MERET PMD 60G-10V0QQ pressure sensor with 0-0,6 MPa range and voltage output. The output of this sensor was fed into analog input of HUMUSOFT MF624 I/O card with sampling period set to $100 \mu$ s. The measurement cycle lasted 25s, with filling valve opened at $t=5 \mathrm{~s}$ and closed at $t=$ $15 \mathrm{~s}$. At $t=19 \mathrm{~s}$ discharge valve was opened and closed at $t=$ 25s (Fig.3).

As is obvious from Fig.3 more significant deviations of the model compared to the real muscle can be observed during the transient responses of muscle filling in contrast to a discharge cycle which is in quite a good accord. Several factors might be responsible for observed discrepancies: the pressure sensor and the muscle are connected with $1 \mathrm{~m}$ long line meaning that the sensor reading has to be corrected; the equation 6 for volume flow might better approximate discharge cycle than a filling cycle; the differential equation 11 makes assumption of isothermal process with ideal gas and it might be needed to refine it; the stiffness coefficient of paralel spring was considered constant. During the experiments, the model was tested in quick-release experiment conditions. Until present day, this test has been carried out only as a simulation (due to the lack of appropriate measuring apparatus) and responses of the model to two releases with $30 \mathrm{~kg}$ load are shown in Fig.4. The muscle was fixed to a zero initial contraction and two different initial pressures and then released. At the beginning, the force of contractile element (as an output of polynomial approximator in Fig.2) is compensated by the force of clamping. At the higher initial force (corresponding to higher initial pressure), the acceleration achieved after release is higher as is the maximum contraction during transients. Relative as well as absolute damping are comparable in both cases.

\section{CONCLUSION}

In this paper a model of pneumatic artificial muscle based on the modified Hill's muscle model used in biomechanics was proposed. Due to the certain similarities between striated

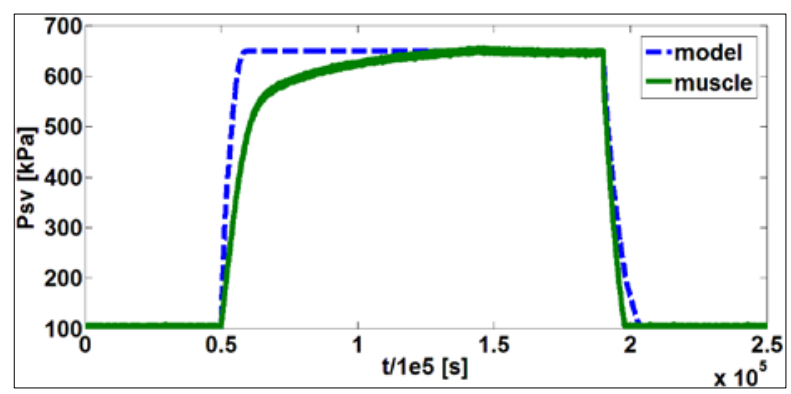

Fig. 3. Responses to a $650 \mathrm{kPa}$ pressure step (dashed - model, full line - real muscle)

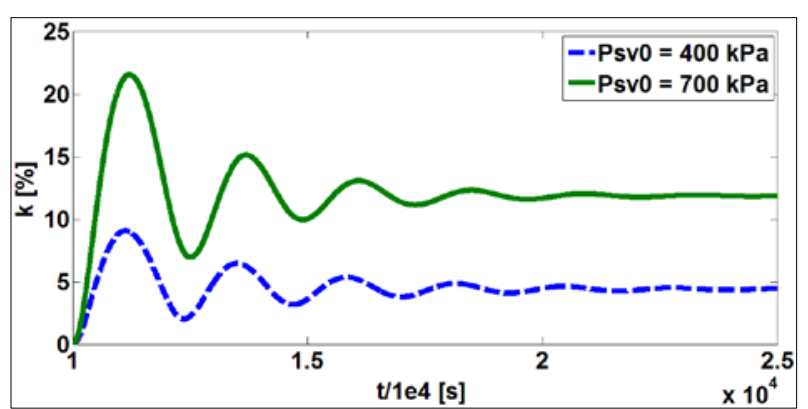

Fig. 4. Quick release simulation for two different initial pressures and zero contraction with $30 \mathrm{~kg}$ load

muscles and artificial muscles, Hill's muscle model seems to be a plausible representation of the properties of artificial muscle and it can be used for their modeling when the unique features are taken into account.

Despite several simplifications, the designed model is able to represent static and dynamic properties of used pneumatic artificial muscle. In further work it will be necessary to concentrate on gathering as much experimental data as possible to be able to truly validate the designed model (finding out the dependencies of stiffness and damping coefficients, improving the accuracy of static force approximator, including pneumatic lines into the model etc.). After validating the model of one muscle, we expect to proceed with proposing the model of dual-muscle actuator for driving a rotational joint.

\section{ACKNOWLEDGEMENTS}

The research work is supported by the Project of the Structural Funds of the EU, Operational Programme Research and Development, Measure 2.2 Transfer of knowledge and technology from research and development into practice. Title of the project: Research and development of nonconventional pneumatic artificial muscles-based actuators. ITMS code: 26220220103

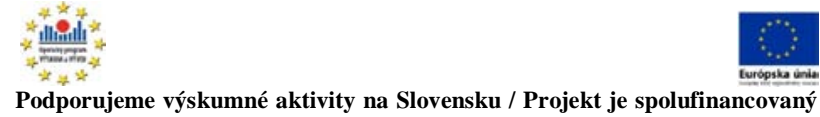
zo zdrojov ES.

\section{REFERENCES}

Beater, P.(2007): Pneumatic Drives. System Design, Modelling and Control. Springer-Verlag, ISBN 3-540-69470-6, Berlin

Boržíková, J.-Pitel'J.(2009): Model of the pneumatic actuator based on artificial muscles, Proceedings of PGP Institute Conference, Pskov, ISBN 9785911160872. pp. 102-104., PGP Institute, Pskov

Kerscher, T. et al.(2005): FLUMUT - Dynamic Modelling of Fluidic Muscles using Quick-Release Experiments Proceedings of 3rd International Symposium on Adaptive Motion in Animals and Machines, Ilmenau, ISBN: 3932633997, TU Ilmenau, Ilmenau

Serres, J.L.(2008): Dynamic Characterization of a Pneumatic Muscle Actuator and Its Application to a Resistive Training Device. Doctoral Thesis. Wright State University Dayton, Ohio (USA). pp.201

Shadmehr, R. - Wise, S.P. (2005): The Computational Neurobiology of Reaching and Pointing. Supplementary Documents. MIT Press, ISBN 0-262-19508-9, Cambridge

Židek, K. et al.(2010): New Trends in Application of Artificial Muscles for Automation Devices in Nonproductive Sector. Vyrobne inzinierstvo. Vol.IX, No.4,2010.pp.78-80,ISSN 1335-7972 